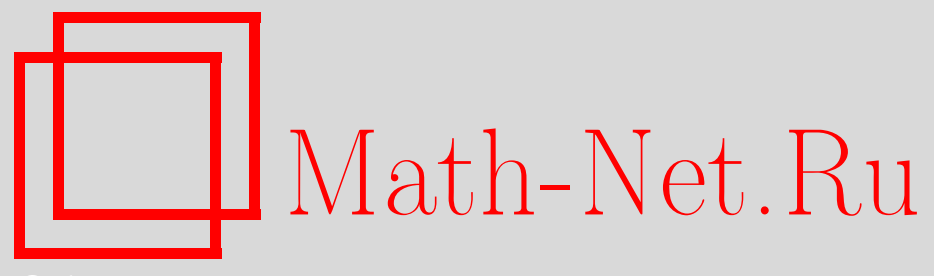

В. Б. Шерстюков, О некоторых признаках полной регулярности роста целых функций экспоненциального типа, Матем. заметки, 2006, том 80, выпуск 1, 119-130

DOI: https://doi.org/10.4213/mzm2787

Использование Общероссийского математического портала Math-Net.Ru подразумевает, что вы прочитали и согласны с пользовательским соглашением http://www . mathnet.ru/rus/agreement

Параметры загрузки:

IP : 54.80 .73 .141

26 апреля 2023 г., 15:46:10

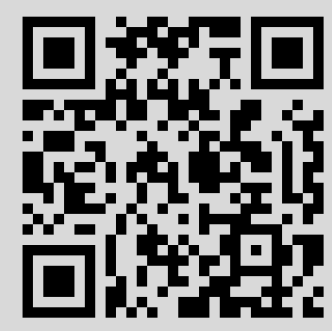




\section{О НЕКОТОРЫХ ПРИЗНАКАХ ПОЛНОЙ РЕГУЛЯРНОСТИ РОСТА ЦЕЛЫХ ФУНКЦИЙ ЭКСПОНЕНЦИАЛЬНОГО ТИПА}

\section{В. Б. Шерстюков}

В заметке усилены предыдущие результаты автора о наличии вполне регулярного роста у целой функции экспоненциального типа с последовательностью простых (всех) нулей $\Lambda=\left\{\lambda_{k}\right\}_{k=1}^{\infty}$. Для функции с вещественными нулями условия регулярности ее роста (на действительной оси и во всей плоскости) выписываются только через оценки снизу на значения модуля производной в точках $\lambda_{k}$. Получен также аналог одной теоремы М.Г. Крейна о функции, обратная величина которой раскладывается в соответствующий ряд простых дробей.

Библиография: 13 названий.

1. Введение. Используемые всюду ниже понятия и факты теории целых функций хорошо известны и содержатся, например, в монографиях [1], [2]. Рассматривается целая функция экспоненциального типа (ЦФЭТ) $\mathscr{L}(\lambda)$, все нули которой простые и образуют последовательность $\Lambda=\left\{\lambda_{k}\right\}_{k=1}^{\infty}\left(\left|\lambda_{1}\right| \leqslant\left|\lambda_{2}\right| \leqslant \cdots\right)$. Пусть $h(\mathscr{L}, \theta)$ - индикатор функции $\mathscr{L}, \theta \in[0,2 \pi)$. В настоящей заметке, служащей продолжением статьи автора [3], изучается следующая общая задача: в какой мере поведение производной $\mathscr{L}^{\prime}(\lambda)$ в точках последовательности $\Lambda$ влияет на регулярность роста самой функции $\mathscr{L}(\lambda)$ ? Исследование этого вопроса инициировано так называемой проблемой А. Ф. Леонтьева [4]: будет ли $\mathscr{L}(\lambda)$ иметь вполне регулярный рост $(\mathrm{BPP})$ во всей плоскости, если выполняется

$$
\varlimsup_{k \rightarrow \infty}\left\{\frac{1}{\left|\lambda_{k}\right|} \ln \frac{1}{\left|\mathscr{L}^{\prime}\left(\lambda_{k}\right)\right|}+h\left(\mathscr{L}, \arg \lambda_{k}\right)\right\} \leqslant 0 ?
$$

Условие (1) и близкие ему по характеру играют важную роль при установлении критериев представимости аналитических функций рядами экспонент или обобщенных экспонент (см., например, [2], [5]) и затрагивают целый ряд смежных вопросов: интерполяции, слабой достаточности ( $\gamma$-достаточности) и максимальности множеств в различных классах целых функций. Из большого количества соответствующих работ упомянем лишь [6]-[9], имеющие некоторое отношение к данной статье. Несмотря на усилия ряда авторов [10], [11], [3], сама задача Леонтьева до сих пор не решена. Чтобы отметить некоторые результаты частного характера, полученные в этом направлении, условимся в обозначениях. Класс всех функций, удовлетворяющих "условию Леонтьева" (1), обозначим через $L$. Определим еще подкласс $L_{0} \subset L$,

(C) В. Б. Шерстюков, 2006 
состоящий из функций с вещественными нулями $\lambda_{k}, k=1,2, \ldots$ В работе [11] А. В. Братищев построил пример четной ЦФЭТ $\mathscr{L}(\lambda) \in L_{0}$, т.е. имеющей вид

$$
\mathscr{L}(\lambda)=\prod_{k=1}^{\infty}\left(1-\frac{\lambda^{2}}{\lambda_{k}^{2}}\right), \quad \lambda_{k} \nearrow+\infty,
$$

которая не является функцией ВРР. В этом примере индикаторная диаграмма $D(\mathscr{L})$ функции $\mathscr{L}$ есть отрезок мнимой оси, поэтому задачу Леонтьева следует решать при дополнительном предположении о наличии внутренних точек у множества $D(\mathscr{L})$. В классе же $L_{0}$ естественной выглядит такая задача: найти условие на последовательность $\left\{\mathscr{L}^{\prime}\left(\lambda_{k}\right)\right\}_{k=1}^{\infty}$, влекущее полную регулярность роста $\mathscr{L}(\lambda)$. Обе эти задачи рассматривались в статье [3], основными результатами которой являются следующие две теоремы.

Tеорема А [3; теорема 2]. Условие

$$
\exists \alpha>0: \frac{1}{\left|\mathscr{L}^{\prime}\left(\lambda_{k}\right)\right|}=O\left(\left|\lambda_{k}\right|^{\alpha}\right), \quad k \rightarrow \infty,
$$

где $\mathscr{L}$ - произвольная ЦФЭТ с простыми нулями $\lambda_{k} \in \mathbb{R}$, выделяет подкласс $L_{1} \subset$ $L_{0}$ такой, что всякая функиия $\mathscr{L} \in L_{1}$ имеет BPP.

Теорема В [3; теорема 3]. Eсли $\mathscr{L} \in L$ имеет положительный индикатор и

$$
\lim _{\substack{\lambda \in S_{\gamma} \\ \lambda \in S_{\gamma}}}|\mathscr{L}(\lambda)|>0
$$

$\left(\gamma\right.$ - некоторая проходящая через начало координат прямая; $S_{\gamma}$ - относительно плотное по мере множество на $\gamma)$, то $\mathscr{L}$ - функиия ВРР.

Там же отмечено, что условие (4) заведомо выполняется, если нижний индикатор функции $\mathscr{L}$

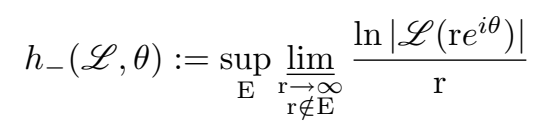

на прямой $\gamma$ положителен (sup берется по множествам E нулевой относительной меры).

Ниже (п. 2, теорема 1) установлены некоторые свойства функций класса $L_{0}$, в частности, полная регулярность роста на вещественной прямой функций вида (2). Этот результат не противоречит цитированному примеру из [11], так как теорема В в этой ситуации, очевидно, неприменима $(\operatorname{int} D(\mathscr{L})=\varnothing)$.

В п. 3 получено усиление теоремы А: в условии (3) под знаком $O$ можно записать $\left|w\left(\lambda_{k}\right)\right|$, где $w(\lambda)$ - целая функция экспоненциального типа нуль $(w(\lambda) \in[1 ; 0])$ со специальными свойствами. Наконец, заключительная часть работы посвящена собственно задаче Леонтьева. В теореме 3 п. 4 приводится ряд новых дополнительных условий на функцию $\mathscr{L} \in L$, при выполнении которых $\mathscr{L}$ - функция ВРР. Одно из них: $\underline{\lim }_{|x| \notin E}|\mathscr{L}(x)| \cdot|x|^{\alpha}>0$ с каким-то $\alpha>0$ и $E^{0}$-множеством $E$ (ср. с неравенством (4) из теоремы В). Обсуждается жесткость этих условий и их независимость. В качестве приложения установлен аналог одной теоремы М.Г. Крейна о разложении обратной величины целой функции класса Картрайт. 
2. Для канонического произведения (2) определяются величины

$$
h(\Lambda):=\varliminf_{k \rightarrow \infty}\left(\lambda_{k+1}-\lambda_{k}\right)
$$

(шаг последовательности $\left.\Lambda=\left\{\lambda_{k}\right\}_{k=1}^{\infty}\right)$ и

$$
\delta(\Lambda):=\varlimsup_{k \rightarrow \infty} \frac{1}{\lambda_{k}} \ln \frac{1}{\left|\mathscr{L}^{\prime}\left(\lambda_{k}\right)\right|}
$$

(индекс конденсации Леонтьева). Как отмечалось выше, функция $\mathscr{L} \in L_{0}$ не обязана иметь во всей плоскости ВРР, однако справедлива

Теорема 1. Индикаторная диаграмма произвольной функиии $\mathscr{L} \in L_{0}$ есть отрезок, параллельный мнимой оси. Далее, четная функиия (2) из класса $L_{0}$ имеет ВРР на вещественной оси и

$$
\delta(\Lambda)=\lim _{k \rightarrow \infty} \frac{1}{\lambda_{k}} \ln \frac{1}{\left|\mathscr{L}^{\prime}\left(\lambda_{k}\right)\right|}=0 .
$$

ДоказАтельство. Считаем, что

$$
\mathscr{L}(\lambda)=e^{a \lambda+b} \cdot \prod_{k=1}^{\infty}\left(1-\frac{\lambda}{\lambda_{k}}\right) e^{\frac{\lambda}{\lambda_{k}}}, \quad a, b \in \mathbb{C} .
$$

Положив $\mathscr{L}_{1}(\lambda):=\mathscr{L}(\lambda) \cdot e^{-(i \operatorname{Im} a) \cdot \lambda}$, получим $\left|\mathscr{L}_{1}(i \mu)\right| \geqslant e^{\operatorname{Re} b} \forall \mu \in \mathbb{R}$. Кроме того,

$$
\begin{aligned}
h\left(\mathscr{L}_{1} ; \frac{\pi}{2}\right) & =\varlimsup_{\mu \rightarrow+\infty} \frac{\ln \left|\mathscr{L}_{1}(i \mu)\right|}{\mu}=\varlimsup_{\mu \rightarrow+\infty} \frac{\ln \prod_{k=1}^{\infty} \sqrt{1+\frac{\mu^{2}}{\lambda_{k}^{2}}}}{\mu} \\
& =\varlimsup_{\mu \rightarrow+\infty} \frac{\ln \left|\mathscr{L}_{1}(-i \mu)\right|}{\mu}=h\left(\mathscr{L}_{1} ; \frac{3 \pi}{2}\right) .
\end{aligned}
$$

Если бы индикаторная диаграмма $D(\mathscr{L})$ имела внутренние точки, то при соответствующем выборе $c \in \mathbb{R}$ мы бы получили $h\left(\mathscr{L}_{2}, \theta\right)>0 \quad \forall \theta$, где $\mathscr{L}_{2}(\lambda):=e^{c \lambda} \cdot \mathscr{L}_{1}(\lambda)$ и $\mathscr{L}_{2}$ удовлетворяет условию (1). По теореме В $\mathscr{L}_{2}$ имеет ВРР и, следовательно, $D\left(\mathscr{L}_{2}\right)$ - отрезок, что противоречит условию $\operatorname{int} D(\mathscr{L}) \neq \varnothing$. Таким образом, $D(\mathscr{L})$ суть отрезок. Индикаторная диаграмма функции $\mathscr{L}$ всегда имеет ось симметрии, параллельную вещественной оси. Поэтому путем параллельного переноса можно добиться того, чтобы $D(\mathscr{L})$ лежала на мнимой оси, что доказывает первую часть теоремы.

Пусть теперь $\mathscr{L} \in L_{0}$ и является четной. По доказанному $h(\mathscr{L} ; 0)=h(\mathscr{L} ; \pi)=$ $h\left(\mathscr{L} ; \arg \lambda_{k}\right)=0$ для всех $k \in \mathbb{N}$. Следовательно, неравенство (1) превращается в равенство

$$
\delta(\Lambda)=\lim _{k \rightarrow \infty} \frac{1}{\lambda_{k}} \ln \frac{1}{\left|\mathscr{L}^{\prime}\left(\lambda_{k}\right)\right|}=0 .
$$

Относительно недавно А. Ю. Поповым было установлено, что

$$
\varlimsup_{k \rightarrow \infty} \frac{1}{\lambda_{k}} \ln \frac{1}{\left|\mathscr{L}^{\prime}\left(\lambda_{k}\right)\right|}=\varlimsup_{x \rightarrow+\infty} \frac{\ln \left(\frac{1}{\mathscr{L}_{0}(x)}\right)}{x},
$$


где $\mathscr{L}_{0}(x)=|\mathscr{L}(x)| / \rho(x), \quad \rho(x)=\inf _{k \geqslant 1}\left|x-\lambda_{k}\right|$, a $\mathscr{L}(\lambda)=\prod_{k=1}^{\infty}\left(1-\lambda^{2} / \lambda_{k}^{2}\right)-$ произвольная четная ЦФЭТ, у которой $\Lambda=\left\{\lambda_{k}\right\}_{k=1}^{\infty}-$ строго возрастающая к $+\infty$ последовательность положительных чисел с шагом $h(\Lambda)$ (доказательство результата при $h(\Lambda)>0$ имеется в работе [12; предложение 2]). Положим $E=$ $\bigcup_{k \in \mathbb{N}}\left[\lambda_{k}-1 / k ; \lambda_{k}+1 / k\right]$ и оценим в условиях теоремы 1 величину $\rho(x)$ снизу на множестве $\mathbb{R}_{+} \backslash E$. Последовательность $\left\{\lambda_{k}\right\}_{k=1}^{\infty}$ имеет конечную верхнюю плотность: $\bar{\Delta}:=\varlimsup_{k \rightarrow \infty} \frac{k}{\lambda_{k}}<+\infty$. В случае $\bar{\Delta}=0$ функция $\mathscr{L}(\lambda) \in[1 ; 0]$ и имеет ВРР во всей плоскости. Пусть $\bar{\Delta}>0$. Для каждого достаточно большого положительного $x \notin E$ найдется номер $n=n(x): \lambda_{n}<x<\lambda_{n+1}$ и при этом $\lambda_{k} / k>1 /(2 \bar{\Delta})$ $\forall k \geqslant n$.

Если $\rho(x)=x-\lambda_{n}$, то

$$
\rho(x)>\frac{1}{n}>\frac{1}{2 \bar{\Delta} \cdot \lambda_{n}}>\frac{1}{2 \bar{\Delta} \cdot x} .
$$

Если же $\rho(x)=\lambda_{n+1}-x$, то

$$
\rho(x)>\frac{1}{n+1}>\frac{1}{2 \bar{\Delta} \cdot \lambda_{n+1}}=\frac{1}{2 \bar{\Delta} \cdot(x+\rho(x))} .
$$

Отсюда $2 \bar{\Delta} \cdot \rho^{2}(x)+2 \bar{\Delta} \cdot x \cdot \rho(x)-1>0$, а значит,

$$
\rho(x)>\frac{-\bar{\Delta} \cdot x+\sqrt{(\bar{\Delta} \cdot x)^{2}+2 \bar{\Delta}}}{2 \bar{\Delta}}=\frac{1}{\bar{\Delta} \cdot x+\sqrt{(\bar{\Delta} \cdot x)^{2}+2 \bar{\Delta}}} .
$$

Итак, $1 / \rho(x)=O(x), x \rightarrow+\infty, x \notin E$, поэтому $\underline{\lim }_{x \rightarrow+\infty} \frac{\ln \rho(x)}{x} \geqslant 0$. Выше установлено, что $\delta(\Lambda)=0$. Это влечет

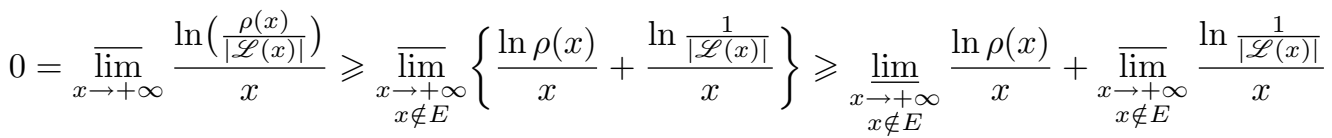

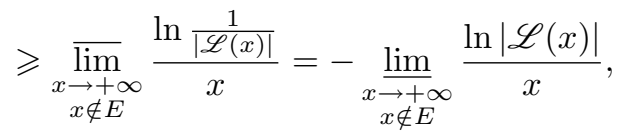

т.e.

$$
\lim _{\substack{x \rightarrow+\infty \\ x \notin E}} \frac{\ln |\mathscr{L}(x)|}{x} \geqslant 0=h(\mathscr{L} ; 0)=\varlimsup_{x \rightarrow+\infty} \frac{\ln |\mathscr{L}(x)|}{x} .
$$

Следовательно, существует обычный $\lim _{x \rightarrow+\infty} \frac{\ln |\mathscr{L}(x)|}{x}=0$. При этом $E$ - множество нулевой относительной меры. В самом деле, обозначив через $N_{\Lambda}(r)=\sum_{\lambda_{k} \leqslant r} 1$ считающую функцию последовательности $\Lambda$, имеем $\overline{\lim }_{r \rightarrow+\infty}\left(N_{\Lambda}(r) / r\right)=\bar{\Delta}$. Далее,

$$
\frac{\operatorname{mes}(E \cap[0, r])}{r} \leqslant \frac{\sum_{k=1}^{N_{\Lambda}(r)} \frac{2}{k}}{r}=\frac{O\left(\ln N_{\Lambda}(r)\right)}{r}=\frac{O(\ln r)}{r}, \quad r \rightarrow+\infty,
$$

поэтому

$$
\lim _{r \rightarrow+\infty} \frac{\operatorname{mes}(E \cap[0, r])}{r}=0 .
$$

Следовательно, четная функция $\mathscr{L}(\lambda)$ имеет $\mathrm{BPP}$ на луче $\arg \lambda=0$. Теорема доказана. 
3. Следующий результат, усиливающий теорему А, дает признак полной регулярности роста ЦФЭТ с простыми вещественными нулями.

Теорема 2. Пусть $w(\lambda)$ - функция класса $[1 ; 0]$, нулевое множество которой имеет вид $\mathscr{N} \cup \mathscr{M}$, где $\mathscr{N}=\left\{\nu_{n}\right\}_{n=1}^{N}, \mathscr{M}=\left\{i \mu_{j}\right\}_{j=1}^{\infty}, \nu_{n} \in \mathbb{C}, \mu_{j} \in \mathbb{R}, n=\overline{1, N} ; j=$ $\overline{1, \infty}$ (одно из множеств $\mathscr{N}$ или $\mathscr{M}$ может отсутствовать). При этом все нули из последовательности $\mathscr{M}$ являются простыми, а $\nu_{n}-$ произвольной кратности $s_{n} \geqslant 1$. Предположим еще (если $\mathscr{M}$ наличествует), что

$$
\exists \alpha>0: \inf _{j \geqslant 1}\left|w^{\prime}\left(i \mu_{j}\right)\right| \cdot\left|\mu_{j}\right|^{\alpha}>0 .
$$

Тогда если $\mathscr{L}(\lambda)$ - ЦФЭТ с простыми вещественными нулями $\left\{\lambda_{k}\right\}_{k=1}^{\infty}: \Lambda \cap \mathscr{N}=\varnothing$, то справедлива импликация: $1 /\left|\mathscr{L}^{\prime}\left(\lambda_{k}\right)\right|=O\left(\left|w\left(\lambda_{k}\right)\right|\right), k \rightarrow+\infty, \Longrightarrow \mathscr{L}$ - функция $\mathrm{BPP}$ и $D(\mathscr{L})$ - отрезок мнимой оси.

Доказательству теоремы 2 предпошлем лемму, имеющую на наш взгляд, и самостоятельный интерес.

Лемма. Пусть $w(\lambda)$ удовлетворяет всем условиям теоремы 2 с заменой условия (5) на более жесткое:

$$
\sum_{j=1}^{\infty} \frac{1}{\left|w^{\prime}\left(i \mu_{j}\right)\right|}<+\infty
$$

Тогда найдется относительно плотное по мере множество $S \subseteq \mathbb{R}$ такое, что

$$
\inf _{\mu \in S}|w(i \mu)|>0 .
$$

ДокАЗАТЕЛьСтво лЕммы. Если нулевое множество функции $w$ содержит $\mathscr{N}$, то, умножив $w$ на соответствующую рациональную функцию, мы получим функцию класса $[1 ; 0]$ с простыми чисто мнимыми нулями, удовлетворяющую (6). Поэтому будем сразу предполагать, что множество $\mathscr{N}$ отсутствует, т.е.

$$
w(\mu)=e^{a \mu+b} \cdot \prod_{j=1}^{\infty}\left(1-\frac{\mu}{i \mu_{j}}\right) e^{\frac{\mu}{i \mu_{j}}}, \quad a, b \in \mathbb{C} .
$$

Введем функцию $w_{0}(\mu):=w(\mu) e^{-\mu \operatorname{Re} a}$. Легко видеть, что $\left|w_{0}(\mu)\right| \geqslant e^{\operatorname{Re} b} \forall \mu \in \mathbb{R}$. Оценим на вещественной оси ЦФЭТ

$$
\varphi(\mu):=\frac{1}{w_{0}(\mu)}-\sum_{j=1}^{\infty} \frac{1}{w_{0}^{\prime}\left(i \mu_{j}\right)\left(\mu-i \mu_{j}\right)} .
$$

Для всех $\mu \in \mathbb{R}$

$$
|\varphi(\mu)| \leqslant \frac{1}{\left|w_{0}(\mu)\right|}+\sum_{j=1}^{\infty} \frac{1}{\left|w_{0}^{\prime}\left(i \mu_{j}\right)\right|\left|\mu-i \mu_{j}\right|} \leqslant e^{-\operatorname{Re} b}+\sum_{j=1}^{\infty} \frac{1}{\left|w_{0}^{\prime}\left(i \mu_{j}\right)\right|\left|\mu_{j}\right|} .
$$

Привлекая (6), получаем ограниченность $|\varphi(\mu)|$ на $\mathbb{R}$. Следовательно, $\varphi(\mu)$ - функция ВРР. Тогда из представления

$$
\varphi(\mu) w_{0}(\mu)=1-\sum_{j=1}^{\infty} \frac{w_{0}(\mu)}{w_{0}^{\prime}\left(i \mu_{j}\right)\left(\mu-i \mu_{j}\right)},
$$


в котором правая часть есть ЦФЭТ $\psi(\mu)$ с $h(\psi ; \theta) \leqslant h\left(w_{0} ; \theta\right)$ для всех $\theta$, выводим $h(\varphi ; \theta) \equiv 0$. Итак, $\varphi \in[1 ; 0]$ и ограничена на $\mathbb{R}$, поэтому $\varphi \equiv c$. Имеем

$$
\frac{1}{w_{0}(\mu)}=c+\sum_{j=1}^{\infty} \frac{1}{w_{0}^{\prime}\left(i \mu_{j}\right)\left(\mu-i \mu_{j}\right)} \quad \text { для всех } \quad \mu \notin \mathscr{M} \text {. }
$$

Положим $S:=\mathbb{R} \backslash \bigcup_{j=1}^{\infty}\left\{\mu \in \mathbb{R}:\left|\mu-\mu_{j}\right|<1\right\}$. Из того, что плотность последовательности $\left\{\mu_{j}\right\}_{j=1}^{\infty}$ равна нулю, немедленно следует, что $S$ относительно плотно по мере на $\mathbb{R} ;$ при этом $\forall \mu \in S$

$$
\frac{1}{\left|w_{0}(i \mu)\right|} \leqslant c+\sum_{j=1}^{\infty} \frac{1}{\left|w_{0}^{\prime}\left(i \mu_{j}\right)\right|}<+\infty,
$$

что доказывает лемму $\left(\left|w_{0}(i \mu)\right|=|w(i \mu)| \forall \mu \in \mathbb{R}\right)$.

ДОКАЖЕм ТЕПЕРЬ ТЕОРЕмУ 2. Введем вспомогательную функцию

$$
w_{1}(\lambda):=\frac{w(\lambda)\left(\lambda^{2 s+1}-1\right)}{\prod_{n=1}^{N}\left(\lambda-\nu_{n}\right)^{s_{n}}} \in[1 ; 0]
$$

с нулями $\mathscr{M} \cup\left\{\xi_{m}\right\}_{m=1}^{2 s+1}$, где $\xi_{m}$ - все значения $\sqrt[2 s+1]{1}$, а $s \in \mathbb{N}: 2 s \geqslant \alpha+\sum_{n=1}^{N} s_{n}+1$, $\alpha$ взято из условия (5). При этом

$$
\frac{1}{\left|w_{1}^{\prime}\left(i \mu_{j}\right)\right|}=O\left(\frac{1}{\left|\mu_{j}\right|^{2}}\right), \quad j \rightarrow+\infty,
$$

что следует из выбора $s$ и условия (5). Поскольку показатель сходимости $\mathscr{M}$ не превосходит 1, то

$$
\sum_{j=1}^{\infty} \frac{1}{\left|w_{1}^{\prime}\left(i \mu_{j}\right)\right|}<+\infty
$$

Таким образом, функция $w_{1}(\lambda)$ удовлетворяет всем требованиям леммы. Найдем по лемме относительно плотное на $\mathbb{R}$ по мере множество $S$ так, чтобы для функции $w_{1}(\lambda)$ выполнялось условие $(7)$. Считая $\mathscr{L}(0) \neq 0$, представим функцию $\mathscr{L}$ в виде

$$
\mathscr{L}(\lambda)=e^{c+d \lambda} \prod_{k=1}^{\infty}\left(1-\frac{\lambda}{\lambda_{k}}\right) e^{\frac{\lambda}{\lambda_{k}}}
$$

и возьмем $\mathscr{L}_{1}(\lambda)=w_{1}(\lambda) e^{-\lambda i \operatorname{Im} d} \mathscr{L}(\lambda)-Ц Ф Э Т$ с простыми нулями $\left\{\xi_{m}\right\}_{m=1}^{2 s+1} \cup \mathscr{M} \cup \Lambda$. Так как $\underline{\lim }_{k \rightarrow \infty}\left|\mathscr{L}^{\prime}\left(\lambda_{k}\right)\right|\left|w\left(\lambda_{k}\right)\right|>0$ и

$$
\frac{1}{\left|w_{1}\left(\lambda_{k}\right)\right|}=O\left(\frac{1}{\left|w\left(\lambda_{k}\right)\right|\left|\lambda_{k}\right|^{\alpha+2}}\right), \quad k \rightarrow \infty,
$$

TO

$$
\begin{aligned}
\frac{1}{\left|\mathscr{L}_{1}^{\prime}\left(\lambda_{k}\right)\right|\left|\lambda_{k}\right|} & =\frac{1}{\left|\lambda_{k}\right|\left|w_{1}\left(\lambda_{k}\right)\right|\left|\mathscr{L}^{\prime}\left(\lambda_{k}\right)\right|} \\
& =O\left(\frac{1}{\left|w\left(\lambda_{k}\right)\right|\left|\lambda_{k}\right|^{\alpha+3}\left|\mathscr{L}^{\prime}\left(\lambda_{k}\right)\right|}\right)=O\left(\frac{1}{\left|\lambda_{k}\right|^{\alpha+3}}\right), \quad k \rightarrow \infty .
\end{aligned}
$$


Отсюда

$$
\sum_{k=1}^{\infty} \frac{1}{\left|\mathscr{L}_{1}^{\prime}\left(\lambda_{k}\right)\right|\left|\lambda_{k}\right|}<+\infty
$$

Далее, из условия (7) для $w_{1}(\lambda)$ выводим $(\mu \in S)$

$$
\frac{1}{\left|\mathscr{L}_{1}(i \mu)\right|}=\frac{e^{-\operatorname{Re} c}}{\left|w_{1}(i \mu)\right| \prod_{k=1}^{\infty} \sqrt{1+\left(\frac{\mu}{\lambda_{k}}\right)^{2}}}=O(1)
$$

т.e.

$$
\inf \left\{\left|\mathscr{L}_{1}(i \mu)\right|: \mu \in S\right\}>0
$$

Положим теперь

$$
a(\lambda):=\sum_{m=1}^{2 s+1} \frac{\mathscr{L}_{1}(\lambda)}{\left(\lambda-\xi_{m}\right) \mathscr{L}_{1}^{\prime}\left(\xi_{m}\right)}+\sum_{j=1}^{\infty} \frac{\mathscr{L}_{1}(\lambda)}{\left(\lambda-i \mu_{j}\right) \mathscr{L}_{1}^{\prime}\left(i \mu_{j}\right)}+\sum_{k=1}^{\infty} \frac{\mathscr{L}_{1}(\lambda)}{\left(\lambda-\lambda_{k}\right) \mathscr{L}_{1}^{\prime}\left(\lambda_{k}\right)}
$$

Заметим, что в силу (6) для $w_{1}$ имеем

$$
\sum_{j=1}^{\infty} \frac{1}{\left|\mathscr{L}_{1}^{\prime}\left(i \mu_{j}\right)\right|}<+\infty
$$

В самом деле, $\left|\mathscr{L}_{1}^{\prime}\left(i \mu_{j}\right)\right|=\left|w_{1}^{\prime}\left(i \mu_{j}\right)\right| e^{\mu_{j} \operatorname{Im} d}\left|\mathscr{L}\left(i \mu_{j}\right)\right| \geqslant e^{\operatorname{Re} c}\left|w_{1}^{\prime}\left(i \mu_{j}\right)\right|, j \geqslant 1$. Привлекая еще $(8)$, установим, что $a(\lambda)-Ц Ф Э Т$ с $h(a ; \theta) \leqslant h\left(\mathscr{L}_{1} ; \theta\right) \quad \forall \theta$. При этом $a\left(\lambda_{k}\right)=$ $a\left(i \mu_{j}\right)=a\left(\xi_{m}\right)=1, k, j \geqslant 1, m=1,2, \ldots, 2 s+1$, откуда $b(\lambda):=(a(\lambda)-1) / \mathscr{L}_{1}(\lambda)-$ ЦФЭТ. Оценим $|b(i \mu)|$ при $\mu \in S$ :

$$
\begin{aligned}
|b(i \mu)| \leqslant & \frac{1}{\left|\mathscr{L}_{1}(i \mu)\right|}+\sum_{m=1}^{2 s+1} \frac{1}{\left|i \mu-\xi_{m}\right|\left|\mathscr{L}_{1}^{\prime}\left(\xi_{m}\right)\right|} \\
& +\sum_{k=1}^{\infty} \frac{1}{\left|i \mu-\lambda_{k}\right|\left|\mathscr{L}_{1}^{\prime}\left(\lambda_{k}\right)\right|}+\sum_{j=1}^{\infty} \frac{1}{\left|\mu-\mu_{j}\right|\left|\mathscr{L}_{1}^{\prime}\left(i \mu_{j}\right)\right|}
\end{aligned}
$$

Первые три слагаемых ограничены сверху на $S$ в силу (8) и (9).

Рассмотрим последнее слагаемое. На множестве $S$ (см. доказательство леммы) $\left|\mu-\mu_{j}\right| \geqslant 1 \forall j \geqslant 1$. Воспользовавшись (10), находим окончательно, что $|b(i \mu)|$ ограничен на $S$. Согласно известному результату Б. Я. Левина (см., например, [13]) $|b(\lambda)|$ ограничен на мнимой оси и, стало быть, $b(\lambda)$ - функция ВРР. Из соотношения $b(\lambda) \mathscr{L}_{1}(\lambda)=a(\lambda)-1$ получаем $b(\lambda) \in[1 ; 0]$, откуда $b(\lambda) \equiv b$. Итак, установлено, что обратная величина целой функции $\mathscr{L}_{1}(\lambda)$ класса Картрайт допускает разложение вида

$$
\frac{1}{\mathscr{L}_{1}(\lambda)}=-b+\sum_{k=1}^{\infty} \frac{a_{k}}{\lambda-\eta_{k}}
$$

в котором $\left\{\eta_{k}\right\}_{k=1}^{\infty}-$ все нули $\mathscr{L}_{1}(\lambda), \sum_{k=1}^{\infty}\left|a_{k}\right| /\left|\eta_{k}\right|<\infty$. По теореме М. Г. Крейна [1; с. 333] $\mathscr{L}_{1}(\lambda)-$ функция $\mathrm{BPP}$ и $D\left(\mathscr{L}_{1}\right)$ - отрезок мнимой оси. Очевидно, $\mathscr{L}(\lambda)$ обладает теми же свойствами. 
ЗАмечаниЕ 1. Нетрудно привести примеры функций $w \in[1 ; 0]$, отличных от многочлена (это будет сделано ниже), удовлетворяющих предположениям доказанной теоремы, и получить для $\left|\mathscr{L}^{\prime}\left(\lambda_{k}\right)\right|$ асимптотические неравенства, влекущие полную регулярность роста функции $\mathscr{L}(\lambda)$ из условия теоремы 2 . Здесь возникает интересная и, по-видимому, трудная задача (на которую любезно обратил внимание автора А.Ю. Попов): довести результат теоремы 2 до неулучшаемого, или, другими словами, указать необходимые и достаточные условия на функцию $\Omega: \mathbb{R} \rightarrow \mathbb{R}_{+}$, обеспечивающие полную регулярность роста всякой ЦФЭТ $\mathscr{L}(\lambda)$ с простыми вещественными нулями $\lambda_{k}$ со свойством $1 /\left|\mathscr{L}^{\prime}\left(\lambda_{k}\right)\right|=O\left(\Omega\left(\lambda_{k}\right)\right), k \rightarrow \infty$. Из цитированного во введении примера Братищева легко усмотреть необходимое условие такого рода: $\underline{\lim }_{r \rightarrow \infty}(\ln \Omega(r)) /|r| \leqslant 0$. Действительно, взяв какую-либо функцию $\Omega: \mathbb{R} \rightarrow \mathbb{R}_{+}$, не удовлетворяющую указанному условию, получим для произвольно зафиксированной последовательности $r=\left\{r_{k}\right\}_{k=1}^{\infty}$ вещественных чисел $\underline{\lim }_{k \rightarrow \infty}\left(\ln \Omega\left(r_{k}\right)\right) /\left|r_{k}\right|=: 2 \beta_{r}>0$. Тогда $e^{\beta_{r}\left|r_{k}\right|}=O\left(\Omega\left(r_{k}\right)\right), k \rightarrow \infty$. Как отмечалось выше (см. введение), существует функция $\mathscr{L} \in L_{0}$ вида (2), не являющаяся функцией ВРР. Тем не менее, равенство

$$
\lim _{k \rightarrow \infty} \frac{1}{\lambda_{k}} \ln \frac{1}{\left|\mathscr{L}^{\prime}\left(\lambda_{k}\right)\right|}=0
$$

дает

$$
\frac{1}{\left|\mathscr{L}^{\prime}\left(\lambda_{k}\right)\right|}=O\left(e^{\beta_{\Lambda} \lambda_{k}}\right)=O\left(\Omega\left(\lambda_{k}\right)\right), \quad k \rightarrow \infty
$$

В то же время достаточное условие выполнения нужной импликации, полученное в теореме 2, содержит неявно неравенство

$$
\varlimsup_{r \rightarrow \infty} \frac{\ln \Omega(r)}{|r|} \leqslant 0 .
$$

Устранение очевидного "зазора" и составляет содержание сформулированной задачи.

Приведем два простых примера функции $w(\lambda)$, удовлетворяющей требованиям теоремы 2 .

ПримеР 1. Пусть $\rho<1$ и $\rho(r)(\rightarrow \rho$ при $r \rightarrow \infty)$ - уточненный по Валирону порядок. Выберем последовательность положительных чисел $\left\{\mu_{j}\right\}_{j=1}^{\infty}$ такую, что $\mu_{j+1}-\mu_{j}>d \mu_{j}^{1-\rho\left(\mu_{j}\right)}$ при $d>0, j \geqslant j_{0}$, и имеющую плотность $\Delta>0$ при порядке $\rho(r)$. Очевидно, функция $w_{1}(\lambda):=\prod_{j=1}^{\infty}\left(1-\lambda^{2} / \mu_{j}^{2}\right) \in[1 ; 0]$. Как известно [2; c. 46-49], при $0<\theta<\pi$

$$
\lim _{r \rightarrow \infty} \frac{\ln \left|w_{1}\left(r e^{i \theta}\right)\right|}{r^{\rho(r)}}=\frac{\pi \Delta \cos \rho\left(\theta-\frac{\pi}{2}\right)}{\sin \frac{\pi \rho}{2}}
$$

и

$$
\lim _{j \rightarrow \infty} \frac{\ln \left|\frac{1}{w_{1}^{\prime}\left(\mu_{j}\right)}\right|}{\mu_{j}^{\rho\left(\mu_{j}\right)}}=-\pi \Delta \operatorname{ctg} \frac{\pi \rho}{2} .
$$

Следовательно, $\exists \sigma>0: \ln \left|w_{1}^{\prime}\left(\mu_{j}\right)\right|>\sigma \mu_{j}^{\rho\left(\mu_{j}\right)} \forall j \geqslant j_{0}$. Функция $w(\lambda):=w_{1}(i \lambda)$ удовлетворяет всем условиям теоремы 2 . 
ПримеР 2. $w_{1}(\lambda)=\prod_{j=1}^{\infty}\left(1-\lambda / j^{2}\right)=\sin \pi \sqrt{\lambda} /(\pi \sqrt{\lambda})$ - целая функция порядка $\rho=1 / 2$, поэтому $w_{1}(\lambda) \in[1 ; 0]$. Легко подсчитать, что $\left|w_{1}^{\prime}\left(\mu_{j}\right)\right|\left|\mu_{j}\right|=1 / 2 \forall j \geqslant 1$, где $\mu_{j}=j^{2}$. Функция $w(\lambda):=w_{1}(i \lambda)$ нужная. Если взять ЦФЭТ $\mathscr{L}(\lambda)$ с вещественными нулями $\left\{\lambda_{k}\right\}_{k=1}^{\infty}$, то асимптотика

$$
\frac{1}{\left|\mathscr{L}^{\prime}\left(\lambda_{k}\right)\right|}=O\left(\left|w\left(\lambda_{k}\right)\right|\right), \quad k \rightarrow \infty,
$$

принимает вид

$$
\frac{1}{\left|\mathscr{L}^{\prime}\left(\lambda_{k}\right)\right|}=O\left(\frac{e^{\pi \sqrt{\frac{\left|\lambda_{k}\right|}{2}}}}{\sqrt{\left|\lambda_{k}\right|}}\right), \quad k \rightarrow \infty,
$$

и влечет (по теореме 2) вполне регулярный рост функции $\mathscr{L}(\lambda)$.

4. В этом пункте изучается вопрос о наличии вполне регулярного роста у функции класса $L$. Сформулируем полученные результаты в виде сводной теоремы.

Теорема 3. Пусть $\mathscr{L} \in L$ и имеет положительный индикатор $h(\mathscr{L} ; \theta)$. Каждое из следующих дополнительных условий обеспечивает $\operatorname{BPP} \mathscr{L}(\lambda)$ :

1) $\exists C>1: \int_{-\infty}^{\infty} \frac{\ln \left(C+\frac{1}{\mid \mathscr{L}_{(x) \mid}}\right)}{1+x^{2}} d x<+\infty$;

2) $\exists \alpha: 0<\alpha<1, \exists E^{0}$-множество $E$, являющееся объединением не более чем счетного числа интервалов с конечной суммой длин, такое, что

$$
\lim _{\substack{x \rightarrow \infty \\|x| \notin E}} \frac{\ln |\mathscr{L}(x)|}{|x|^{\alpha}}>-\infty
$$

3) $\underline{\lim }_{|x| \notin E}|\mathscr{L}(x)||x|^{\alpha}>0 \quad(\alpha$ u $E-$ как в условии 2$\left.)\right)$;

4) обратная величина функиии $\mathscr{L}(\lambda)$ допускает представление

$$
\frac{1}{\mathscr{L}(\lambda)}=\sum_{k=1}^{\infty} \frac{a_{k}}{\lambda-\lambda_{k}}, \quad \sum_{k=1}^{\infty}\left|a_{k}\right|<\infty ;
$$

ряд сходится поточечно на множестве $\mathbb{C} \backslash \Lambda$.

ЗАмЕчание 2. Условия 1)-4), конечно, не являются независимыми. В самом деле, возможность разложения

$$
\frac{1}{\mathscr{L}(\lambda)}=\sum_{k=1}^{\infty} \frac{a_{k}}{\lambda-\lambda_{k}}, \quad\left\{a_{k}\right\}_{k=1}^{\infty} \in \ell^{1},
$$

в котором ряд простых дробей сходится в каждой точке $\lambda \notin \Lambda$, позволяет сразу оценить снизу $|\mathscr{L}(\lambda)|$ вне фиксированного множества кружков нулевой линейной плотности. Во-первых, из поточечной сходимости ряда $\sum_{k=1}^{\infty} a_{k} /\left(\lambda-\lambda_{k}\right)$ на $\mathbb{C} \backslash \Lambda$ и условия $\sum_{k=1}^{\infty}\left|a_{k}\right|<\infty$ следует его равномерная сходимость внутри области $\mathbb{C} \backslash \Lambda$. Пусть последовательность чисел $r_{m}>0$ такова, что окружности $\gamma_{m}=\{\lambda \in \mathbb{C}$ : $\left.\left|\lambda-\lambda_{m}\right|=r_{m}\right\}, m \in \mathbb{N}$, не пересекаются и внутри $\gamma_{m}$ нет других точек последовательности $\Lambda$, кроме $\lambda_{m}$. Тогда

$$
\frac{1}{\mathscr{L}^{\prime}\left(\lambda_{m}\right)}=\frac{1}{2 \pi i} \int_{\gamma_{m}} \frac{d \lambda}{\mathscr{L}(\lambda)}=\sum_{k=1}^{\infty} \frac{a_{k}}{2 \pi i} \int_{\gamma_{m}} \frac{d \lambda}{\lambda-\lambda_{k}}=a_{m}, \quad m=1,2, \ldots
$$


Предположим, что $\left\{k^{2} / \mathscr{L}^{\prime}\left(\lambda_{k}\right)\right\}_{k=1}^{\infty} \in \ell^{1}$ (это, очевидно, выполняется для функции $\mathscr{L} \in L$ c $h(\mathscr{L}, \theta)>0)$. Из условия 4) получим $\forall \lambda \notin U=\bigcup_{k=1}^{\infty}\left\{\lambda \in \mathbb{C}:\left|\lambda-\lambda_{k}\right|<1 / k^{2}\right\}$ цепочку неравенств

$$
\frac{1}{|\mathscr{L}(\lambda)|} \leqslant \sum_{k=1}^{\infty} \frac{\left|a_{k}\right|}{\left|\lambda-\lambda_{k}\right|} \leqslant \sum_{k=1}^{\infty} \frac{k^{2}}{\left|\mathscr{L}^{\prime}\left(\lambda_{k}\right)\right|}=: \frac{1}{C}>0 .
$$

Обозначим $E_{1}=-\left(\mathbb{R}_{-} \cap U\right) ; E_{2}=\mathbb{R}_{+} \cap U ; E=E_{1} \cup E_{2}$. Вне $E^{0}$-множества $E(|x| \notin E)$ на $\mathbb{R}$ выполняется неравенство $|\mathscr{L}(x)| \geqslant C$, откуда подавно имеем $\lim _{\substack{x \rightarrow \notin E \\|x| \notin}}|\mathscr{L}(x)||x|^{\alpha}=+\infty \quad \forall \alpha>0$. Итак, в предположениях теоремы 34$\left.) \Rightarrow 3\right)$. Проверим теперь, что для произвольной ЦФЭТ $\mathscr{L}(\lambda)$ выполнено 3$) \Rightarrow 2) \Rightarrow 1)^{\prime}$. Здесь 1)' получается из 1$)$, если интегрирование вести по множеству $\mathbb{R} \backslash E, E=\bigcup_{k=1}^{\infty}\left(\alpha_{k}, \beta_{k}\right)$, $\sum_{k=1}^{\infty}\left(\beta_{k}-\alpha_{k}\right)<\infty$. Неравенство 3) влечет

$$
\lim _{\substack{x \rightarrow \infty \\|x| \notin E}} \frac{\ln |\mathscr{L}(x)|}{|x|^{\alpha}} \geqslant 0>-\infty .
$$

Далее, из 2) получим при некотором $\sigma>0$ и всех $|x| \notin E 1 /|\mathscr{L}(x)| \leqslant e^{\sigma|x|^{\alpha}}$.

Тогда при $C=e^{\sigma}>1$

$$
\begin{aligned}
\int_{\mathbb{R} \backslash E} \frac{\ln \left(C+\frac{1}{|\mathscr{L}(x)|}\right)}{1+x^{2}} d x & \leqslant \int_{\mathbb{R} \backslash E} \frac{\ln \left(C+e^{\sigma|x|^{\alpha}}\right)}{1+x^{2}} d x<\int_{-\infty}^{+\infty} \frac{\ln \left(e^{\sigma}+e^{\sigma|x|^{\alpha}}\right)}{1+x^{2}} d x \\
& =2\left[\int_{0}^{1} \frac{\ln \left(e^{\sigma}+e^{\sigma x^{\alpha}}\right)}{1+x^{2}} d x+\int_{1}^{+\infty} \frac{\ln \left(e^{\sigma}+e^{\sigma x^{\alpha}}\right)}{1+x^{2}} d x\right] \\
& \leqslant 2\left[\int_{0}^{1} \frac{\ln \left(2 e^{\sigma}\right)}{1+x^{2}} d x+\int_{1}^{+\infty} \frac{\ln \left(2 e^{\sigma x^{\alpha}}\right)}{1+x^{2}} d x\right] \\
& =2\left[\ln 2 \int_{0}^{+\infty} \frac{d x}{1+x^{2}}+\sigma \int_{0}^{1} \frac{d x}{1+x^{2}}+\sigma \int_{1}^{+\infty} \frac{x^{\alpha}}{1+x^{2}} d x\right] \\
& <\pi \ln 2+\sigma\left(\frac{\pi}{2}+\frac{2}{1-\alpha}\right)=: M_{\mathscr{L} .}
\end{aligned}
$$

Заключение теоремы 3 , как будет следовать из приводимого ниже доказательства, остается в силе, если заменить условие 1) на 1)'․ Поскольку само доказательство почти не претерпевает изменений, то теорему 3 , таким образом, достаточно установить при более естественном, нежели 1$)^{\prime}$, дополнительном предположении 1). Само условие 1) полезно сравнить с одним известным неравенством, имеющим место для произвольной ЦФЭТ [1; с. 296]:

$$
\exists M_{\mathscr{L}}<+\infty: \forall R>0 \quad \int_{0}^{R} \frac{\ln \frac{1}{|\mathscr{L}(x)| \cdot|\mathscr{L}(-x)|}}{1+x^{2}} d x<M_{\mathscr{L}} .
$$

Известно также (частный случай теоремы 6 [7]), что всякая ЦФЭТ $\mathscr{L}$ с простыми нулями $\left\{\lambda_{n}\right\}_{n=1}^{\infty}$ и такая, что $\underline{\lim }_{k \rightarrow \infty} 1 /\left|\lambda_{k}\right| \ln \left|\mathscr{L}^{\prime}\left(\lambda_{k}\right)\right|>-\infty$, удовлетворяет вне некоторой системы попарно непересекающихся кружков $\left|\lambda-\lambda_{k}\right|<r_{k}, \quad \sum_{k=1}^{\infty} r_{k}<$ $+\infty$, оценке: $\exists C_{1}, C_{2}>0$ : $|\mathscr{L}(\lambda)| \geqslant C_{1} \cdot \exp \left\{-C_{2}|\lambda|\right\}$, из которой легко следует:

$$
2)^{\prime} \quad \lim _{\substack{x \rightarrow \infty \\|x| \notin E}} \frac{\ln |\mathscr{L}(x)|}{|x|}>-\infty
$$


для некоторого $E^{0}$-множества $E$ (ср. с условием 2)).

Сформулируем, наконец, в виде отдельного утверждения содержащийся в теореме 3 аналог цитированного выше (см. доказательство теоремы 2) результата М. Г. Крейна о разложении обратной величины целой функции класса Картрайт.

СлЕДСТВИЕ. Пусть обратная величина ЦФЭТ $\mathscr{L}$ с простыми нулями $\left\{\lambda_{k}\right\}_{k=1}^{\infty} u$ положительным индикатором $h(\mathscr{L} ; \theta)$ допускает разложение "типа М. Г. Крейна":

$$
\frac{1}{\mathscr{L}(\lambda)}=\sum_{k=1}^{\infty} \frac{a_{k}}{\lambda-\lambda_{k}}, \quad \lambda \in \mathbb{C} \backslash \Lambda,
$$

коэббициенты а которого подчинены неравенству

$$
\varliminf_{k \rightarrow \infty}\left\{\frac{1}{\left|\lambda_{k}\right|} \ln \left|a_{k}\right|-h\left(\mathscr{L}, \arg \lambda_{k}\right)\right\} \geqslant 0 .
$$

Тогда $\mathscr{L}$ - функиия ВРР.

ДокАЗАТЕЛЬСтво тЕоремы 3. Следуя схеме доказательства теоремы В (см. [3; с. 307-309]), возьмем выпуклую область $G$ с опорной функцией $h(-\theta)$ и рассмотрим аналитическую в $G$ (при фиксированном $\lambda \in \mathbb{C}$ ) функцию

$$
\psi(\lambda, z)=\frac{e^{\lambda z}}{\mathscr{L}(\lambda)}-\sum_{k=1}^{\infty} \frac{e^{\lambda_{k} z}}{\mathscr{L}^{\prime}\left(\lambda_{k}\right)\left(\lambda-\lambda_{k}\right)} .
$$

В [3] отмечено, что $\psi(\lambda, z)$ является по $\lambda$ ЦФЭТ (если зафиксировать $z \in G$ ). Кроме того, можно подобрать лежащий в $G$ отрезок $K$, числа $\delta>0$ и $A>1$ так, чтобы для всех вещественных $x$ вне объединения $U$ кружков $U_{k}=\left\{\lambda:\left|\lambda-\lambda_{k}\right|<e^{-\delta\left|\lambda_{k}\right|}\right\}$ и всех $z \in K$ выполнялось неравенство

$$
|\psi(x, z)| \leqslant \frac{1}{|\mathscr{L}(x)|}+\sum_{k=1}^{\infty} \frac{e^{\left|\lambda_{k}\right|\left(h_{K}\left(\arg \lambda_{k}\right)+\delta\right)}}{\left|\mathscr{L}^{\prime}\left(\lambda_{k}\right)\right|} \leqslant \frac{1}{|\mathscr{L}(x)|}+A .
$$

Здесь $h_{K}(-\theta)$ обозначает опорную функцию компакта $K$. Можно считать, что $A \leqslant$ $C$ (при $A>C$ все последующие рассуждения проходят для функции $(C / A) \psi(x, z))$. Покажем, что $\forall z \in K \exists C_{z}<\infty$ :

$$
\int_{-\infty}^{+\infty} \frac{\ln _{+}|\psi(x, z)|}{1+x^{2}} d x \leqslant C_{z}
$$

Из (11) следует

$$
\ln _{+}|\psi(x, z)| \leqslant \ln \left(\frac{1}{|\mathscr{L}(x)|}+A\right) \leqslant \ln \left(\frac{1}{|\mathscr{L}(x)|}+C\right) \quad \forall z \in K, \quad \forall x \in \mathbb{R} \backslash U .
$$

На множестве $\mathbb{R} \cap U$ (если оно не пусто) функция $\psi(\lambda, z)$ удовлетворяет оценке $\ln _{+}|\psi(x, z)| \leqslant \sigma_{z} \cdot|x| \quad\left(0<\sigma_{z}<+\infty \quad \forall z \in K\right)$. Поскольку $\sum_{k=1}^{\infty} e^{-\delta\left|\lambda_{k}\right|}<+\infty$ и $|x| /\left(1+x^{2}\right) \leqslant 1 / 2 \forall x \in \mathbb{R}$, то, привлекая условие 1$)$ теоремы 3 , получаем нужное. Согласно теореме Картрайт [1; с. 315] при каждом $z \in K \psi(\lambda, z)$ в силу (12) является 
(по $\lambda$ ) функцией вполне регулярного роста. Последнее обстоятельство позволяет из представления $(z \in K)$

$$
\psi(\lambda, z) \mathscr{L}(\lambda)=e^{\lambda z}-\sum_{k=1}^{\infty} \frac{\mathscr{L}(\lambda) e^{\lambda_{k} z}}{\mathscr{L}^{\prime}\left(\lambda_{k}\right)\left(\lambda-\lambda_{k}\right)}
$$

вывести $\forall \theta \in[0,2 \pi)$ следующее неравенство для индикаторов: $h_{z}(\psi, \theta)+h(\mathscr{L}, \theta) \leqslant$ $h(\mathscr{L}, \theta)$. Итак, $\forall z \in K \quad \psi(\lambda, z) \in[1 ; 0]$ и поэтому $\forall \epsilon>0 \exists B_{z, \epsilon}<+\infty:|\psi(\lambda, z)| \leqslant$ $B_{z, \epsilon} e^{\epsilon|\lambda|} \forall \lambda \in \mathbb{C}$. Отрезок $K$ можно взять таким: $[-i a, i a], a>0$. Положив в (13) $z=i a, \lambda=-i x, x \in \mathbb{R}_{+} \backslash U$, имеем с некоторыми константами $B_{a}, C_{a}<+\infty$ неравенство $B_{a} e^{\frac{a}{2} x}|\mathscr{L}(-i x)| \geqslant e^{a x}-C_{a}|\mathscr{L}(-i x)|$, откуда $|\mathscr{L}(-i x)| \geqslant D_{a} e^{a x / 2} \forall x \in \mathbb{R}_{+} \backslash U$. Аналогичное неравенство $|\mathscr{L}(i x)| \geqslant \widetilde{D}_{a} e^{\frac{a}{2} x} \quad \forall x \in \mathbb{R}_{+} \backslash U$, выводится из (13) при $z=-i a$. Значит, на мнимой оси имеется относительно плотное по мере множество $S$, для которого $\lim _{\lambda \rightarrow \infty}|\mathscr{L}(\lambda)|=+\infty$. Доказательство заканчивается применением теоремы В.

\section{СПИСОК ЦИТИРОВАННОЙ ЛИТЕРАТУРЫ}

[1] Б. Я. Левин, Распределение корней целых функций, Гостехиздат, М., 1956.

[2] А.Ф. Леонтьев, Ряды экспонент, Наука, М., 1976.

[3] В.Б. Шерстюков, "Об одной задаче Леонтьева и представляющих системах экспонент", Матем. заметки, 74:2 (2003), 301-313.

[4] А.Ф. Леонтьев, "Об условиях разрешимости аналитических функций в ряды Дирихле", Изв. АН СССР. Сер. матем., 36:6 (1972), 1282-1295.

[5] Ю. Ф. Коробейник, "Представляющие системы", УМН, 36:1 (1981), 73-126.

[6] А.В. Абанин, Достаточные множества и абсолютно представляющие системъ, Дисс. ... д.ф.-м.н., Екатеринбург, 1995.

[7] А. В. Братищев, "Один тип оценок снизу целых функций конечного порядка и некоторые приложения", Изв. АН СССР. Сер. матем., 48:3 (1984), 451-475.

[8] Ю.Ф. Коробейник, "Максимальные и $\gamma$-достаточные множества. Приложения к целым функциям. 2", Теория функций, функционалъный анализ и их приложения, 55, Харьков, 1991, 23-34.

[9] В.Б. Шерстюков, "К вопросу о $\gamma$-достаточных множествах", Сиб. матем. ж., 41:4 (2000), 935-943.

[10] Ю. И. Мельник, "О представлении регулярных функций рядами типа рядов Дирихле", Исследование по теории приближений функиий и их приложения, Киев, 1978, $132-141$.

[11] А. В. Братищев, "К одной задаче А. Ф. Леонтьева", Докл. АН СССР, 270:2 (1983), 265-267.

[12] А. Ю. Попов, “Точная оценка индекса конденсации”, Mathematica Montisnigri, 11 (1999), 67-103.

[13] Б. Я. Левин, "Почти периодические функции с ограниченным спектром", Актуальные вопросы математического анализа, Изд-во РГУ, Ростов-на-Дону, 1978, 112-124.

В. Б. Шерстюков

Московский инженерно-физический институт 